\title{
Optical Trapping and Propagation of Nonresonantly Driven One-Dimensional Exciton-Polariton Condensate
}

\author{
A. Opala*, M. Pieczarka And G. SęK \\ Laboratory for Optical Spectroscopy of Nanostructures, Department of Experimental Physics, \\ Faculty of Fundamental Problems of Technology, Wrocław University of Science and Technology, \\ Wyb. S. Wyspiańskiego 27, 50-370 Wrocław, Poland
}

\begin{abstract}
We study theoretically a nonresonant optical creation of a one-dimensional exciton-polariton condensate in a semiconductor microcavity. The polariton condensate is treated in the mean-field approach, taking into consideration an antitrapping potential created by the reservoir of noncondensed particles. Polariton condensates are excited by multiple lasers, with a combination of continuous wave and pulsed sources. The proposed pump-probe configuration leads to the realisation of various experimental schemes, e.g. optical trapping of a polariton condensate in real space. Moreover, it can be utilised for investigation of elementary excitations in the time domain when polariton condensates from two sources interact with each other.
\end{abstract}

DOI: 10.12693/APhysPolA.132.401

PACS/topics: 67.85.De, 71.36.+c, 03.75.Kk

\section{Introduction}

Exciton-polaritons are bosonic light-matter quasiparticles with extremely low effective mass (between 0 characteristic for a photon and the mass of an exciton) and strong exciton-mediated interparticle interactions [1]. They can be formed as a superposition of a photon mode and a quantum well exciton achieved in a strong coupling regime, where due to finite lifetime of a photon confined inside a microcavity, polariton condensates need to be externally pumped, e.g. via nonresonant laser.

Modern semiconductor technology allows the realisation of patterned photonic nanostructures of an arbitrary design [2]. Using the state-of-the-art etching techniques one can prepare structures enabling photon and exciton confinement to be effectively one-dimensional [3]. This kind of structures can be also found in a naturally formed defect in a planar microcavity $[4,5]$. Additional condensate patterning can be achieved using the nonlinear properties of nonresonantly excited exciton-polaritons, where repulsive interactions create an optically induced potential [6-8]. Moreover, multiple laser sources can provide a scheme to study dynamics of quantum coherence of polariton condensates [9].

In this contribution, we propose a pump-probe type of experiment with two excitation sources which create two separate reservoirs and condensates in different positions of one-dimensional polariton microwire. We calculate the time dynamics within the mean-field approach in various pump configurations.

\footnotetext{
* corresponding author; e-mail: andrzej.opala@student.pwr.edu.pl
}

\section{Model}

We study the dynamics of an exciton-polariton macroscopic wave function $\psi(x t)$ using a mean-field theory, solving numerically open-dissipative one-dimensional Gross-Pitaevskii equation (GPE). The GPE is coupled to the rate equation for the incoherent polariton reservoir $n_{R}(x t)[8]:$

$$
\begin{aligned}
& \mathrm{i} \hbar \frac{\partial \psi(x, t)}{\partial t}=\left(-\hbar^{2} \nabla_{x}^{2} / 2 m^{*}+V_{a t}(x, t)\right. \\
& \left.\quad+\frac{\mathrm{i} \hbar}{2}\left(R^{1 D} n_{R}(x, t)-\gamma_{C}\right)\right) \psi(x t), \\
& \frac{\partial n_{R}(x, t)}{\partial t}=P(x t) \\
& \quad-\left(\gamma_{R}+R^{1 D}|\psi(x, t)|^{2}\right) n_{R}(x t) .
\end{aligned}
$$

where $m^{*}$ is the lower polariton effective mass, $V_{a t}$ represents an antitrapping potential (described below), $R^{1 D}$ is a rate of the stimulated scattering, $P$ is a pumping rate which describes the reservoir injection in time and space. We assume that stimulated relaxation of polaritons from reservoir to the condensate is described by a linear term $R^{1 D} n_{R}(x t)$. Additionally, we consider finite lifetime of polaritons $\left(\tau_{C}\right)$ and the reservoir, described by loss rates $\gamma_{C}=\frac{1}{\tau_{C}}$ and $\gamma_{R}=\frac{1}{\tau_{R}}$. In our case $\gamma_{R} \gg \gamma_{C}$, which fulfills the adiabatic condition in reservoir relaxation [10]. The constants $g_{C}$ and $g_{R}$ describe nonlinear interactions in system. Here $g_{C}$ characterize interactions between polaritons in the condensate and $g_{R}$ between condensate and the incoherent exciton reservoir. It should be noted that in our experimental configuration, where polaritons are confined in one-dimensional semiconductor quantum wire, the system parameters must be rescaled when compared to the two-dimensional case [10] $\left(R^{1 D}, g_{i}^{1 D}\right)=\left(R^{2 D}, g_{i}^{2 D}\right) / \sqrt{2 \pi d^{2}}$ ( $d$ stands for the width of the microwire and $i=\{C R\}$ ). In the present work we used the following parameters [8]: $m_{p}=0.42 \frac{\mathrm{meV} \mathrm{ps}^{2}}{\mu \mathrm{m}^{2}}$, 
$\gamma_{C}=0.76 \frac{1}{\mathrm{ps}}, \gamma_{R}=3.04 \frac{1}{\mathrm{ps}}, g_{C}=0.03 \frac{\mu \mathrm{m}^{2}}{\mathrm{ps}}, g_{R}=$ $0.06 \mathrm{meV} \mu \mathrm{m}^{2}, R=0.1 \frac{\mu \mathrm{m}^{2}}{\mathrm{ps}}, P_{t h}=23.32 \frac{\mu \mathrm{m}^{2}}{\mathrm{ps}}, L=$ $300 \mu \mathrm{m}, D=4 \mu \mathrm{m}$. The initial state in the simulation is a white Gaussian noise $d \psi$ in a polariton and exciton field. The simulation is started in absence of polariton steady state: $\psi(x, 0)+d \psi \ll \psi_{0}(x, t)=\sqrt{\frac{P(x, t)-P_{t h}}{\gamma_{C}}}$, where $\psi_{0}(x, t)$ is a stationary state for homogeneous pumping case and $P_{t h}$ is the condensation threshold $P_{t h}=\frac{\gamma_{C} \gamma_{R}}{R}$.

We consider a semiconductor microcavity whose length is measured in hundreds of $\mu \mathrm{m}$ (e.g. $L=300 \mu \mathrm{m}$ ) and width in single $\mu \mathrm{m}$ (e.g. $D=4 \mu \mathrm{m})$, achievable in modern semiconductor processing techniques. Polaritons are generated optically using a non-resonant excitation provided by two sources. One of the sources (pump) provides the steady state local excitation and the second one (probe) creates a dynamical pulse, which creates a pulse of a second condensate, see Fig. 1.

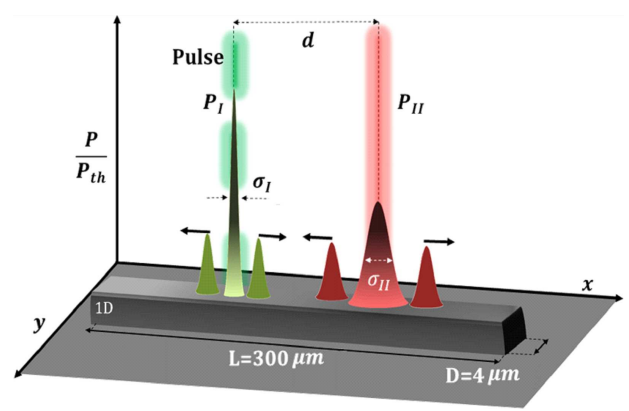

Fig. 1. Scheme of the proposed experimental setup. Details are given in the main text.

The pump source is depicted in Fig. 1 by a red streak and is a single mode continuous wave (CW) laser focused to a larger size spot of $15 \mu \mathrm{m}$ width. The green streak in Fig. 1 represents an ultrashort laser excitation probe pulse, which generates a second polariton condensate. The probe pulse is focused to a $2 \mu \mathrm{m}$ diameter spot and its temporal full width at half maximum is $5 \mathrm{ps}$. The laser excitations creates a local reservoir density described by a pump profile, which generates the local effective antitrapping potential dependent of reservoir and condensate density and strength of the pump fields [8].

The polariton-polariton and polariton-exciton interaction is effectively repulsive $\left(g_{c}^{1 D} g_{R}^{1 D}>0\right)$ [11] and the reservoir and polaritons create an antitrapping potential. This potential can be treated phenomenologically by the following expression:

$$
\begin{aligned}
& V_{a t}(x, t)=\hbar g_{C}^{1 D}|\psi(x, t)|^{2} \psi(x t) \\
& \left.\quad+\hbar g_{R}^{1 D} n_{R}(x t) \psi(x t)+\hbar G P(x t)\right) .
\end{aligned}
$$

In our model, the excitation sources are $P(x, t)=$ $P_{\text {pulse }}(x, t)+P_{C W}(x, t)$, where $P$ is a sum of Gaussian pulse $P_{\text {Pulse }}(x t)$ and continuous laser beam $P_{C W}(x t)$. As a result, interaction between polaritons in $V_{a t}$ causes quasiparticles to be ballistically accelerated away from the potential centre located within the laser source [8],
Fig. 1. The additional repulsive potential strength caused by the pump's field is described by phenomenological coefficient $G(>0)$ which can be determined experimentally, by measuring the emission peak blueshift [8]. Under a non-resonant excitation, photons from continuous laser and a pulse laser generate a population of free electrons and holes with high energy, which contributes to the potential via $\hbar G P(x t)$. Subsequently they relax and form the reservoir and scatter to the condensate. The spatial (and temporal for laser pulse) shape of the laser beams $P_{\text {Pulse }}, P_{C W}$ are described by Gaussian functions

$$
\begin{aligned}
& P_{P u l s e}(x t)=P_{I} \mathrm{e}^{-\left(\frac{x-x_{I}}{\sigma_{S I}^{2}}\right)} \mathrm{e}^{-\left(\frac{t-t_{I}}{\sigma_{T I}^{2}}\right)}, \\
& P_{C W}(x t)=P_{I I} \mathrm{e}^{-\left(\frac{x-x_{I I}}{\sigma_{S I I}^{2}}\right)},
\end{aligned}
$$

where $\sigma_{S I}, \sigma_{S I I}$ and $\sigma_{T I I}$ are the spatial and temporal widths of pumping functions respectively, $x_{I}$ and $x_{I I}$ are the centres of the excitation sources. $P_{I}$ and $P_{I I}$ are the sources amplitudes. For description of this system we prepared a numerical scheme based on time-splitting spectral methods [12] with periodic boundary conditions. These conditions are sufficient for simulations when polariton density vanishes at the end of the numerical box, therefore we have chosen the spatial dimensions to be much larger than the condensate size. In our considerations, we used one-dimensional GPE. Theoretical justification for this model is based on confinement of polaritons in one dimension within the semiconductor microwire. In this case the polariton condensate can flow only in one direction (along the microwire). Parameter $D$ characterizing the width of the microwire is used to rescale the parameters of the model. This approach is based on recent works in the literature $[8,10,13]$.

\section{Results}

At first, we describe the polariton condensate dynamics created by a single laser source. In Fig. $2 \mathrm{a}$ and $\mathrm{b}, \mathrm{a}$ time evolution of a condensate generated by a continuous wave Gaussian pump is presented. In this case, we consider a pump spot: $\sigma_{S I I}=15 \mu \mathrm{m}$ and $P_{I I}=10 P_{t h}$. As it is shown in Fig. 2, in the long time scale, the system tends to be a dynamically stable steady state. In this type of excitation, polaritons are ballistically accelerated from the pump spot. The free polariton flight is represented by two intense peaks in the momentum space distribution, see Fig. 2b.

In the second case, we consider a narrow pulse excitation with intensity profile calculated by $\sigma_{S I}=2.0 \mu \mathrm{m}$, $P_{I}=80 P_{t h}$ and temporal full width half maximum $\sigma_{T I}=5$ ps, Fig. 2c and d. As it can be seen, the excitation generates a short pulse in the polariton density. Polaritons flow away from the centre of the pump spot with specified finite wave-vector, Fig. 2d. Narrow short pulse causes a local reservoir density and effective high antitrapping potential, which lasts shortly in time. This is reflected in a broad spatial distribution of the polariton condensate, Fig. 2c. Combination of these two methods 

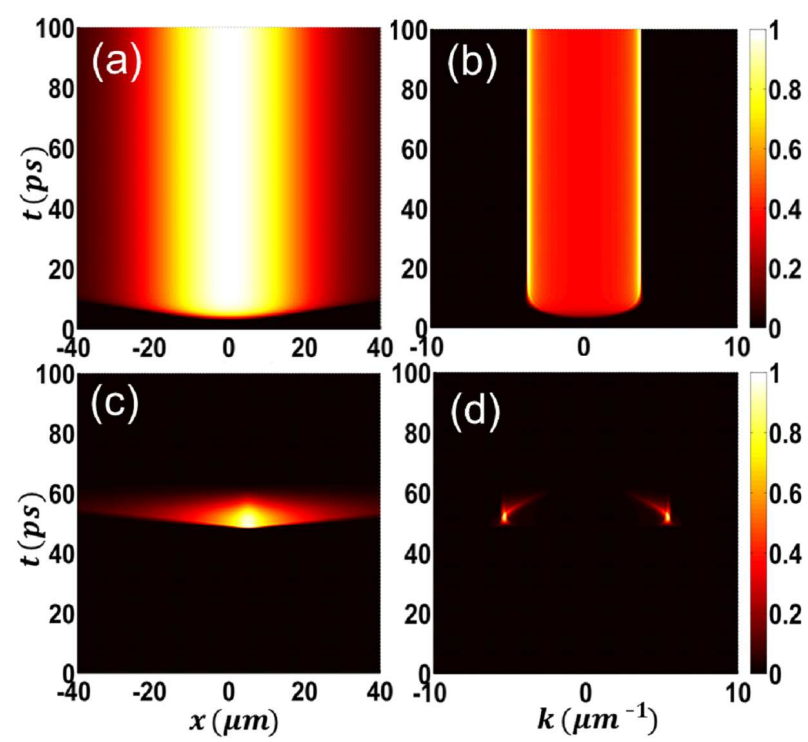

Fig. 2. Time evolution of a CW condensate in (a) real and (b) momentum space. Used parameters are $\sigma_{S I I}=15 \mu \mathrm{m}, P_{I I}=10 P_{t h}$. Time evolution of a pulsed condensate in (c) real and (d) momentum space, where the parameters are $\sigma_{S I}=15 \mu \mathrm{m}, P_{I}=10 P_{t h}$, $\sigma_{T 1}=5$ ps.
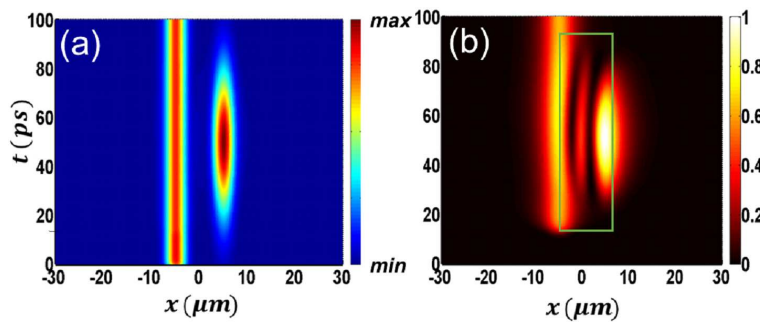

Fig. 3. A trapped polariton configuration. (a) Relative antitrapping potential generated during realisation. (b) Wave-function density time evolution. Confined mode is indicated by a green rectangle. Parameters: $\sigma_{S I}=$ $2 \mu \mathrm{m}, P_{I}=15 P_{t h}, \sigma_{T 1}=20 \mathrm{ps}, x_{I}=5 \mu \mathrm{m}, \sigma_{S I I}=$ $2 \mu \mathrm{m}, P_{I I}=5 P_{t h}, x_{I}=-5 \mu \mathrm{m}$.

of excitation is the basis of the pump-probe setup proposed here.

To generate an effective trapping potential one has to place two abovementioned excitation sources relatively close to each other, as presented in Fig. 3a, where we consider a slightly longer pulse $P_{\text {pulse }}(x t)$. This is close to a real-world realisations of polariton condensates dynamics which are dependent on the internal relaxation of electron-hole pairs [14]. One can observe that in the middle, a dynamical confined mode is formed between the two condensates created at the positions of laser spots (pump and probe), Fig. 3b. Dynamical change in the $P_{\text {pulse }}(x t)$ generated a potential which causes the trapped mode movement, as indicated in Fig. 3b. Additional change in the pump strength or shape can modify the
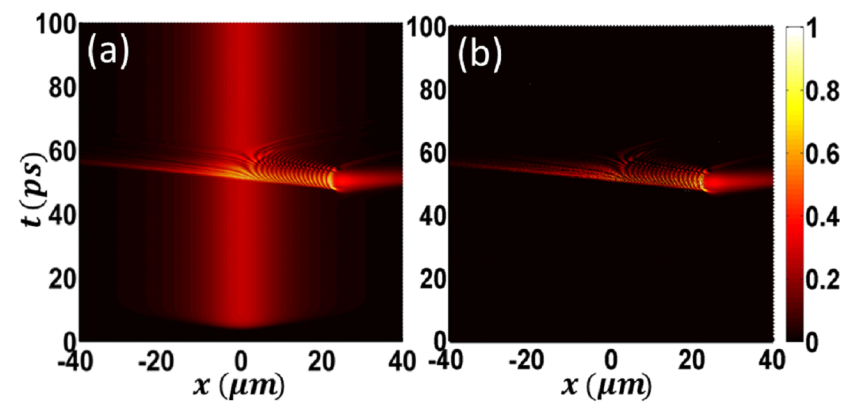

Fig. 4. Proposal of experimental realisation of excitations of a condensate. (a) Time evolution of condensate scattering using two sources. (b) The same evolution, but with subtracted CW condensate, which can be measured in a separate experiment. Parameters: $\sigma_{S I}=10 \mu \mathrm{m}, P_{I}=125 P_{t h}, \sigma_{T 1}=2 \mathrm{ps}, x_{I}=25 \mu \mathrm{m}$, $\sigma_{S I I}=5 \mu \mathrm{m}, P_{I I}=10 P_{t h}$.

confined mode to realise any desired configuration.

An interesting pumping scheme can be obtained, when two laser spots $P_{P u l s e}(x t)$ and $P_{C W}(x t)$ are separated by a larger distance in space, see Fig. 4a. Here, the CW pump source is much weaker than the pulsed source, which is placed tens of $\mu \mathrm{m}$ away. One can observe that the propagating polaritons from the pulsed probe source are scattered by the second condensate, which is visible as an interference pattern in Fig. 4a. This scattering can potentially be the source of excitations of a polariton condensate to the Bogoliubov branches $[15,16]$. In order to detect the change in a polariton wave generated by two sources, one can measure the time dynamics separately using, e.g. a streak camera. Then, two measurements can be subtracted from each other and the result can be analysed in terms of a change in the polariton wave-function dynamics, for example shown in Fig. 4b.

\section{Conclusions}

In conclusion, we used a mean-field theory to investigate the exciton-polariton dynamics under non-resonant pumping. A pump-probe configuration is proposed for studying dynamical properties of interactions between two separate condensates. We presented a scheme for realisation of a dynamical trapping of a polariton condensate and method of investigation of scattering between two condensates. We suppose that this experimental configuration enables a generation of nonlinear excitations like dark solitons or to study elementary superfluid excitations of a polariton condensate. Furthermore, a precise adjustment of the two beams might even lead to generation of dark soliton trains [13], when the $P_{P u l s e}(x t)$ can create a potential step in the condensate generating nonlinear excitations. We hope that this will motivate the future theoretical and experimental research on such multi beam generation of condensates, their interaction and dynamics. 


\section{References}

[1] I. Carusotto, C. Ciuti, Rev. Mod. Phys. 85, 299 (2013).

[2] C. Schneider, K. Winkler, M.D. Fraser, M. Kamp, Y. Yamamoto, E.A. Ostrovskaya, S. Höfling, Rep. Prog. Phys. 80, 16503 (2017).

[3] E. Wertz, L. Ferrier, D. Solnyshkov, R. Johne, D. Sanvitto, A. Lemaître, I. Sagnes, R. Grousson, A.V. Kavokin, P. Senellart, G. Malpuech, J. Bloch, Nat. Phys. 6, 19 (2010).

[4] J. Cuadra, D. Sarkar, L. Vińa, J.M. Hvam, A. Nalitov, D. Solnyshkov, G. Malpuech, Phys. Rev. B 88, 235312 (2013).

[5] F. Manni, K.G. Lagoudakis, B. Pietka, L. Fontanesi, M. Wouters, V. Savona, R. André, B. DeveaudPlédran, Phys. Rev. Lett. 106, 176401 (2011).

[6] A. Askitopoulos, H. Ohadi, A.V. Kavokin, Z. Hatzopoulos, P.G. Savvidis, P.G. Lagoudakis, Phys. Rev. B 88, 41308 (2013).

[7] X. Ma, O.A. Egorov, S. Schumacher, Phys. Rev. Lett. 118, 157401 (2017).

[8] M. Wouters, I. Carusotto, C. Ciuti, Phys. Rev. B 77, 115340 (2008).
[9] C. Antón, G. Tosi, M.D. Martín, Z. Hatzopoulos, G. Konstantinidis, P.S. Eldridge, P.G. Savvidis, C. Tejedor, L. Vińa, Phys. Rev. B 90, 081407(R) (2014).

[10] N. Bobrovska, M. Matuszewski, Phys. Rev. B 92 35311 (2015).

[11] B. Deveaud-Plédran, Comp. Rend. Phys. 1, 1 (2016).

[12] X. Antoine, W. Bao, C. Besse, Comput. Phys. Commun. 184, 2621 (2013).

[13] F. Pinsker, H. Flayac, Phys. Rev. Lett. 112, 140405 (2014)

[14] C. Antón, T.C.H. Liew, G. Tosi, M.D. Martín, T. Gao, Z. Hatzopoulos, P.S. Eldridge, P.G. Savvidis, L. Vińa, Phys. Rev. B 88, 35313 (2013).

[15] M. Pieczarka, M. Syperek, L. Dusanowski, J. Misiewicz, F. Langer, A. Forchel, M. Kamp, C. Schneider, S. Hoefling, A. Kavokin, G. Sek, Phys. Rev. Lett. 115, 186401 (2015).

[16] V. Kohnle, Y. Léger, M. Wouters, M. Richard, M.T. Portella-Oberli, B. Deveaud-Plédran, Phys. Rev. Lett. 106, 255302 (2011). 